\title{
Third order Bragg grating filters in small sol waveguides
}

\section{Seong Phun Chan}

Vittorio M. N. Passaro

passaro@deemail.poliba.it

Goran Z. Mashanovich

Graham Ensell

Graham T. Reed
Intel Technology Sdn. Bhd., Assembly Technology Development Malaysia (ATD-M), but carried out this work whilst at the University of Surrey

Politecnico di Bari, Dipartimento di Elettrotecnica ed Elettronica, via Edoardo Orabona n. 4, 70125 Bari, Italy

The Advanced Technology Institute, University of Surrey, Guildford, Surrey, UK, GU2 7XH

Innos Ltd., Southampton, UK, SO17 1BJ

The Advanced Technology Institute, University of Surrey, Guildford, Surrey, UK, GU2 7XH

Third order grating filters fabricated in small Silicon-on-Insulator rib waveguides are demonstrated. Variations in grating etch depth and duty cycle are considered, and a maximum experimental reflection of $42 \%$ is demonstrated for gratings of $1500 \mu \mathrm{m}$ in length, with a grating period of approximately $689 \mathrm{~nm}$ and an etch depth of $200 \mathrm{~nm}$. Agreement with modeling is shown to be good. [D0I: 10.2971/je0s.2007.07029]

Keywords: Fabrication, filters, integrated optics, optical waveguides, rib waveguides, third order gratings

\section{INTRODUCTION}

Wavelength filters are essential components in modern longhaul and metropolitan optical fiber wavelength division multiplexing (WDM) communication systems. The need to lower system costs is driving a trend towards increased integration of optical devices into more compact subsystems with higher functionality. Waveguide-based integrated-optical technologies are being developed to address this requirement. In particular, grating-assisted waveguide filters are important, since they offer a number of advantages compared to conventional implementations in photosensitive optical fibers. Bragg gratings in integrated optical devices can be realized using processes such as doping, implantation or various etching processes where the strength of the refractive index perturbation could be significantly higher than achieved in fiber, giving more compact devices. Integrated gratings can contain precise phase shifts and variations in grating strength to achieve a desired filter response. In addition, one can realize Bragg gratings in non-photosensitive material such as silicon, which provides mature processing technology and potential monolithic integration with other optical components such as modulators, couplers, or arrayed waveguide gratings to form integrated functionality in a single optical chip.

Grating filters in periodically corrugated rib waveguides have been shown to exhibit high extinction ratios over a narrow band of frequencies [1]. Their reflection response can be tailored by apodization of the grating structure [2]. Using rib waveguide structures as the design platform, the effect of polarisation on the waveguide can be minimized or controlled with appropriate waveguide height to width ratio. Several examples of Bragg grating devices in SOI have been reported using different fabrication methods such as e-beam lithography [3, 4], focused ion beam (FIB) milling [5], femtosecond annealing of amorphous silicon [6], and forming polysilicon/silicon gratings [2]. As Bragg gratings in silicon require a submicron grating period (less than $250 \mathrm{~nm}$ for a wavelength around $1550 \mathrm{~nm}$ ), more relaxed fabrication resolution (with reduced cost) is allowed by third order gratings. In this paper, fabrication and characterisation of third order gratings on SOI rib waveguides is presented for the first time to the best of our knowledge. Moreover, experimental results are compared with Floquet-Bloch Theory (FBT) theoretical predictions [7]. In fact, FBT is known to be a very accurate numerical method to predict the properties and performance of a number of grating devices. Differently from other more popular approaches, such as coupled mode theory (CMT) or transfer matrix method (TMM), FBT takes precisely into account both the optical field distribution and the optical losses as induced by the grating presence. This result is obtained by a rigorous series expansion of the field in space harmonics, whose phase is related to each other by the Floquet condition [7]. Several devices have been studied by FBT, including grating-assisted directional couplers, photonic bandgap crystals and vertical cavity surface emitting lasers.

\section{RESULTS}

Devices were fabricated in SOI waveguides, having a rib height of $1.36 \mu \mathrm{m}$, etch depth of $0.86 \mu \mathrm{m}$ and rib width of $1.00 \mu \mathrm{m}$. The shallow Bragg gratings were confined to the top of the SOI waveguides using a dual mask fabrication pro- 
cess [4]. These Bragg gratings have a period in the range $0.689-0.700 \mu \mathrm{m}$ and etch depth in the range $180-200 \mathrm{~nm}$.

The experimental results for 3rd order Bragg gratings in small SOI waveguides are discussed and presented in comparison with theoretical simulation results. To facilitate the measurement of propagation loss of the waveguides, both the facets of the straight SOI rib waveguides were polished in order to minimize scattering loss from the waveguide facet. A FabryPerot (FP) cavity is formed between the input and the output of the waveguide as a result of the polishing procedure. Subsequently, the propagation loss of the waveguide was evaluated using the FP resonance method [1], yielding unexpectedly high loss of approximately $4.8 \mathrm{~dB} / \mathrm{cm}$ for TE polarization, which may be due to relatively rough waveguide sidewalls, which are sometimes significantly higher for small rib waveguide dimensions.

The reflection experiment for the third order grating devices was performed by using an Agilent 81640A tunable laser source (TLS) $(1520-1620 \mathrm{~nm})$ as the input optical source which was connected to a polarization maintaining tapered lensed fiber, in turn coupled into the SOI rib waveguides. The reflected optical signals were collected via an optical circulator connected to the input of the tapered lensed fiber and recorded by an optical power meter module. The experimental setup is shown in Figure 1. All the experiments were conducted using TE polarization. In order to obtain the reflectiv-

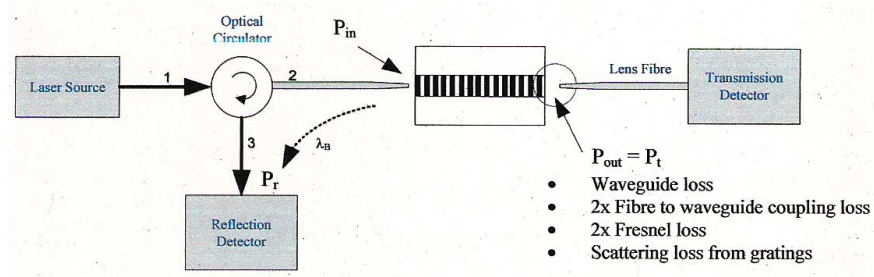

FIG. 1 The experiment schematic layout for the evaluation of Bragg gratings reflectivity.

ity from the 3rd order Bragg gratings, the input power range $(0.66-0.83 \mathrm{~mW})$ from the tunable laser source and the insertion loss of the optical circulator $(1.29-1.55 \mathrm{~dB})$ were investigated in the region of $1540-1560 \mathrm{~nm}$ operating wavelengths. The transmission measurement of the gratings was essential to act as a normalizing factor for the evaluation of Bragg grating reflectivity. The output power provided by the transmission spectrum at non resonance enabled an estimate of the signal reflected by the Bragg grating at the input of tapered lensed fiber. It is assumed that the transmission spectrum includes the propagation loss of the waveguide, two fiber to waveguide coupling losses and Fresnel losses, and scattering loss from the gratings.

The reflection spectra for two 3rd order Bragg gratings with an etch depth of $200 \mathrm{~nm}$ and a $1500 \mu \mathrm{m}$ grating length over the $1540-1560 \mathrm{~nm}$ wavelength range exhibited the characteristic Bragg resonance peak wavelengths, approximately $\lambda_{B}=1553 \mathrm{~nm}$ with full width at half maximum (FWHM) of $3.75 \mathrm{~nm}$ and sidelobe suppression of $8 \mathrm{~dB}$ for a grating period of $0.689 \mu \mathrm{m}$, and $\lambda_{B}=1569 \mathrm{~nm}$ and FWHM of $3 \mathrm{~nm}$ for a grating period of $0.697 \mu \mathrm{m}$, respectively. Note that the reflected powers is relatively low, less than $10 \mu \mathrm{m}$. This is due to the high fiber to waveguide coupling loss to small SOI waveguides, the scattering loss induced by the Bragg gratings and the high waveguide loss. To further explain the low optical power, we estimate the losses introduced during the experiment as follows. The overlap integral between input fiber to waveguide modes was evaluated by the beam propagation method (BPM) to calculate a theoretical coupling efficiency of approximately $56 \%$ for the best case scenario. Assuming Fresnel reflection between silicon and air interface as 0.3 , the measured SOI waveguide propagation loss was $4.8 \mathrm{~dB} / \mathrm{cm}$ at TE polarization while the input power was $0.78 \mathrm{~mW}$ at $1553 \mathrm{~nm}$. The theoretical transmitted power of $14.7 \mu \mathrm{W}$ was calculated based on the aforementioned assumption. The discrepancy between the measured transmission (average $4.5 \mu \mathrm{W}$ ) and theoretical values is explained by deviations of alignment between fiber to waveguide coupling and grating scattering losses, of the order of $3.2 \mathrm{~dB} / \mathrm{mm}$ as calculated by FBT.

In order to compare between the theory and experimental results, both SEM and AFM analysis were used to study the Bragg grating surface features such as grating period and etch depth. The SEM analysis was performed using an FEI Quanta 200F, whereas AFM analysis was conducted using a Digital Instrument Dimension 3100, both at the Advanced Technology Institute, University of Surrey.

Figure 2 depicts the waveguide dimensions of the SOI waveguides on one of the functional devices which exhibited good reflection characteristics. The $\mathrm{SiO}_{2}$ layer on top of the SOI waveguide structure featured in Figure 2 was subsequently stripped and removed by buffered HF in order to reveal the grating structures hidden beneath it (see Figure 3). The thermal isolation trenches which are approximately $6 \mu \mathrm{m}$ from the rib waveguide, are clearly visible from the SEM image.

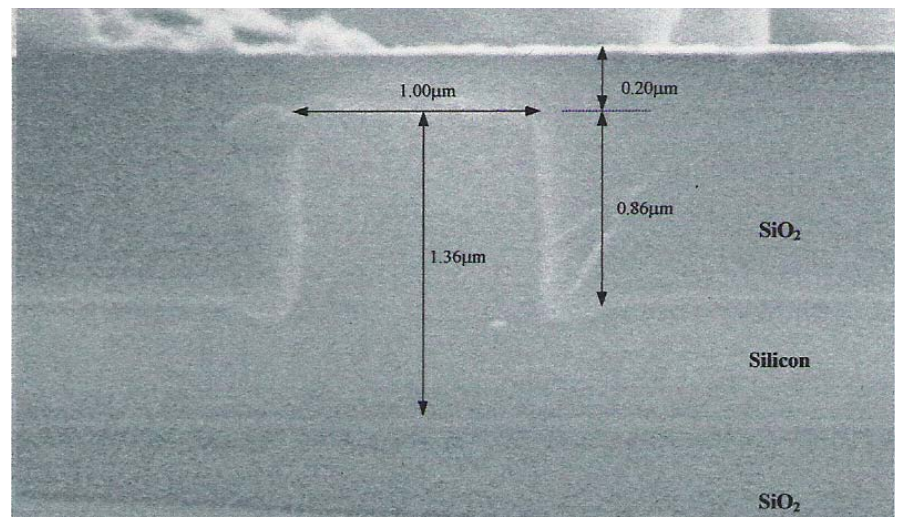

FIG. 2 SEM image of fabricated SOI rib waveguide structure with $1.36 \mu \mathrm{m}$ in height, etch depth of $0.86 \mu \mathrm{m}$ and width of $1.00 \mu \mathrm{m}$. The top $\mathrm{SiO}_{2}$ cladding layer is approximately 20onm from the top of the waveguide.

As the designed and fabricated Bragg grating devices are working in 3rd order, FBT simulations suggest that the maximum reflectivity achievable is smaller than that for the 1st order grating with the same refractive index contrast, grating depth and grating length.

The measured maximum reflectivity is 0.42 for a grating 


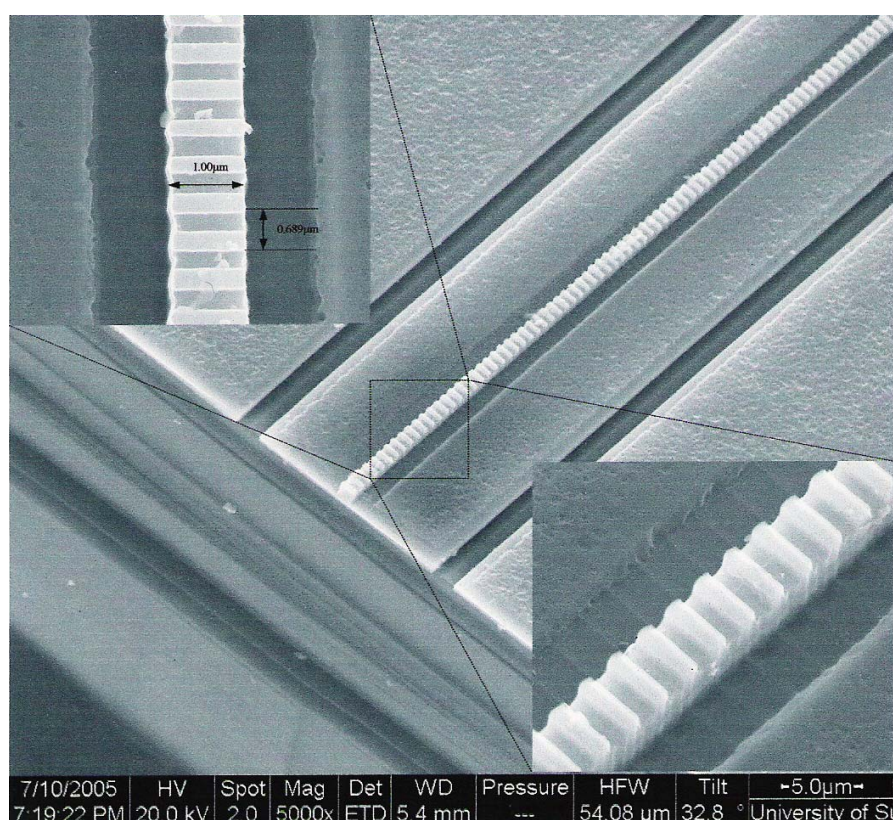

FIG. 3 SEM images of fabricated gratings with grating periods of $0.689 \mu \mathrm{m}$ view at 32.8 tilt angle after the top cladding $\mathrm{SiO}_{2}$ layer was removed by buffered $\mathrm{HF}$. The close up image of the gratings revealed that the gratings are confined only on top of the rib.

length of $1500 \mu \mathrm{m}$ and grating period of approximately $0.689 \mu \mathrm{m}$. The corresponding grating and waveguide dimensions determined in previous SEM inspections were used to simulate the spectral response by FBT with different grating duty cycles and these are presented on the same figure for comparison purposes.

Figure 4 shows a plot of the calculated and measured Bragg resonance wavelengths as a function of grating period variation for $200 \mathrm{~nm}$ etch depth. The Bragg resonance wavelength exhibits a linear relationship with grating period, and shows excellent agreement between calculated and measured result, albeit for only 3 data points.

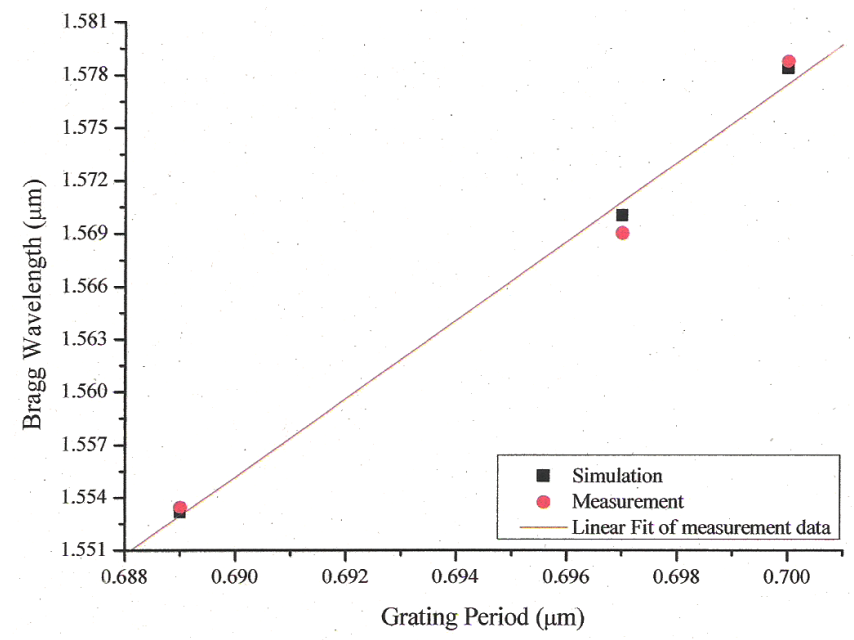

FIG. 4 Bragg wavelength, $\lambda_{B}$ shows a linear shift as a function of grating period for simulation and measurement results, while the line is regression fit.

Finally, Figure 5 shows the influence of duty cycle on the maximum reflectivity and FWHM of grating. Good agreement between the FBT calculations and the measured 3rd order Bragg gratings is shown with $50 \%$ duty cycle, $180 \mathrm{~nm}$ etch depth and approximately $0.700 \mu \mathrm{m}$ grating period, using a quasi-2D approach for FBT and taking into account the scattering loss. The increment of duty cycle (from $50 \%$ to $58 \%$ ) reduces the maximum reflectivity achievable by the grating etch, at the same time narrowing the spectral response of the gratings (due to decreased grating strength), and shifting the peak wavelength at larger values, as expected.

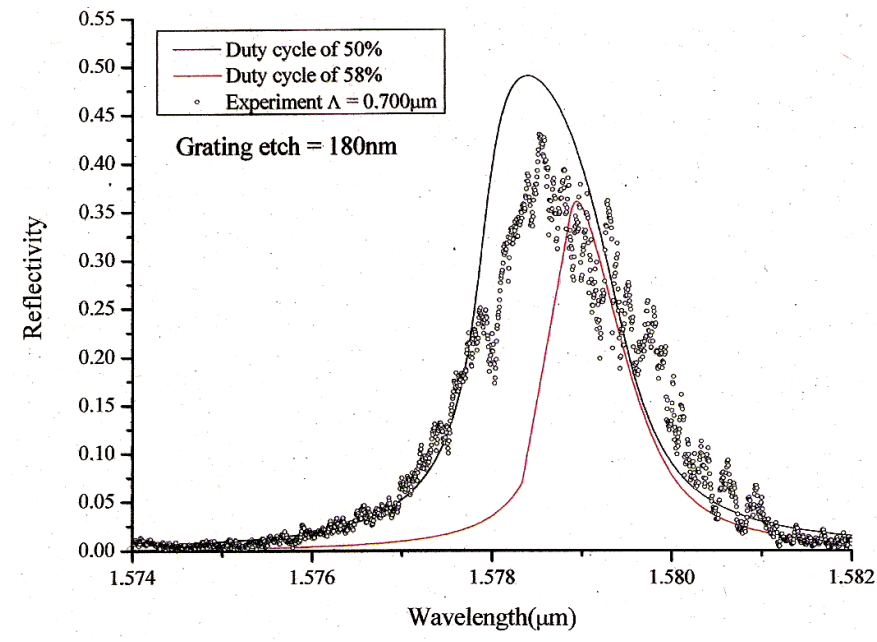

FIC. 5 Reflection spectral response for 3rd order Bragg grating with $1500 \mu \mathrm{m}$ grating length, etch depth of $180 \mathrm{~nm}$ and grating period of $0.700 \mu \mathrm{m}$ (solid lines: FBT predictions).

\section{CONCLUSION}

We have demonstrated the potential of 3rd order Bragg gratings on SOI rib waveguides with grating periods in the region of $0.700 \mu \mathrm{m}$, length of $1500 \mu \mathrm{m}$, and depth of $180-200 \mathrm{~nm}$. The gratings were fabricated by the dual-mask process. The maximum peak reflectivity (42\%) attainable by variation of grating etch depth on the 3rd order Bragg gratings indicates good agreement with the prediction of Floquet-Bloch Theory, when the depth of the periodic corrugation of the grating induces strong perturbation. The variation of duty cycle in the gratings has a significant impact on broadening the spectral response and changing the Bragg conditions of the grating, leading to the shift of Bragg resonance wavelength. Moreover, a linear dependence of Bragg wavelength on the grating period has been demonstrated at constant etch depth by both experiments and FBT modeling. These devices could find applications for out-coupling to SOI waveguides and for filtering in multiplexing applications by appropriately controlling the grating duty cycle. In fact, the relevant FWHM less than $1 \mathrm{~nm}$ should allow the ITU L-band wavelength grid requirement for dense WDM to be satisfied, but with much more relaxed fabrication constraints. Further applications could be also achieved by grating apodization or chirping, such as filtering with strong side lobe suppression.

\section{ACKNOWLEDGMENTS}

The authors wish to thank the University of Delaware for providing a test sample. This work was partially supported by 
Italian Ministry for University and Research under Interlink Project n. II04C01CDM.

\section{References}

[1] G. T. Reed, A. P. Knights, Silicon Photonics (John Wiley at Sons, 2004).

[2] L. Liao, A. Liu, S. Pang, M. J. Paniccia, “Tunable Bragg grating filters in SOI waveguides" OSA Technical Digest on Integrated Photonics Research, IThE2 (2004).

[3] T. Chu, H. Yamada, S. Ishida, and Y. Arakawa, "Tunable optical add-drop multiplexer based on silicon photonic wire waveguides" IEEE Photonic. Tech. L. 18, 1409-1411 (2006).
[4] T. E. Murphy, J. T. Hastings, H. I. Smith, "Fabrication and characterisation of narrow-band Bragg-reflection filters in Silicon-onInsulator ridge waveguides" J. Lightwave Technol. 19, 1938-1942 (2001).

[5] D. J. Moss, V. G. Ta'eed, B. J. Eggleton, D. Freeman, S. Madden, M. Samoc, B. Luther-Davies, S. Janz, and D.-X. Xu, "Bragg gratings in silicon-on-insulator waveguides by focused ion beam milling" Appl. Phys. Lett. 85, 4860-4862 (2004).

[6] G. J. Lee, J. Park, E. K. Kim, Y. P. Lee, K. M. Kim, H. Cheong, C. S. Yoon, Y. D. Son, and J. Jang, "Microstructure of femtosecond laserinduced grating in amorphous silicon" Opt. Express 13, 6445-6453 (2005).

[7] V. M. N. Passaro, "Optimal design of grating-assisted directional couplers" J. Lightwave Technol. 18, 973-984 (2000). 